Artigo Original

\title{
Caracterização do perfil da violência sexual em crianças e adolescentes no Rio Grande do Sul
}

Profile characteristics of sexual violence in children and teenagers in Rio Grande do Sul

Carolina Barbosa Silva ${ }^{1}$, Bibiana Lopes De A. Bastos ${ }^{1}$, Camila Ribeiro Santos ${ }^{1}$, Michele Da Silva Moraes ${ }^{1}$, Lia Gonçalves Possuelo $^{1}$

\section{RESUMO}

${ }^{1}$ Programa de Pós Graduação em Promoção da Saúde

2 Docente do do Programa de Pós Graduação em Promoção da Saúde

UNISC, Santa Cruz do Sul, RS, Brasil

Justificativa e objetivos: A violência sexual infantil é uma das causas de maior morbidade e mortalidade infantil no Brasil e no mundo, onde foi reconhecida como crime desde a década de 80, a partir da Constituição e Estatuto da criança e do adolescente. Considerada uma experiência traumática na infância ou adolescência que reflete em atos para toda vida, tanto físicos como psicológicos, visto pela sociedade como agentes transgressores dos direitos da infância. Este estudo tem como objetivo caracterizar o perfil da violência sexual contra crianças e adolescentes no estado Rio Grande do Sul. Métodos: Trata se de um estudo transversal, descritivo, com dados coletados de base de dados secundária (DATASUS), no período de 2011 a 2015. Resultados: Os resultados mostram que as vítimas mais vulneráveis estão entre os 5 e 9 anos (30,64\%) e 10 a 14 anos (39,35\%), predominantemente no sexo feminino. Os perfis de agressores que se destacaram foram os amigos/conhecidos e familiares como pai e padrasto, sendo a casa das vítimas o local de ocorrência que mais evidenciou. Conclusão: Isso mostra que deve ser melhorada a notificação destes casos, para que se possa gerar punição aos agressores e mais proteção às crianças e adolescentes do estado.

DESCRITORES: violência sexual, abuso sexual de crianças e adolescentes e notificações de abuso.

ABSTRACT: Background and objectives: Child sexual abuse is a cause of increased morbidity and mortality in Brazil and worldwide, which was recognized as a crime since the 80's, from the Constitution and Statute child and adolescent. Considered a traumatic experience in childhood or 
adolescence reflecting on actions for life, both physical and psychological, seen by society as transgressors agents of children's rights. Methods: This is a descriptive study, with data collected from secondary database in the period 2011 to 2015 and aims to characterize the profile of sexual violence in the RS. Results: The results show that the most vulnerable victims are between 5 to 9 $(30,64 \%)$ and 10 to 14 years $(39,35 \%)$ predominantly in females. The profiles of offenders who stood out were the friends / acquaintances and family as father and stepfather, the house of the victims and the place of occurrence that more evident. Conclusion: This shows that the notification of these cases should be improved so that it can generate punishment for offenders and more protection to children and adolescents from the state. KEYWORDS: sexual violence, sexual abuse of children and adolescents and abuse notifications.

\section{INTRODUÇÃO}

A violência sexual infantil é uma das maiores causas de morbidade e mortalidade em crianças e adolescentes no mundo, e é considerada crime que constitui um problema de Saúde Pública. A Organização Mundial da Saúde (OMS) define violência sexual como uma ação incompreendida, e não consentida, devido a sua imaturidade física e emocional devido ao importante envolvimento da criança ou adolescente. Trata-se de um assunto delicado e silencioso, pois nem sempre é notificado, sendo considerado como omissão, o que dificulta a busca de dados nos Sistemas Nacional de Agravos e Notificações (SINAN). ${ }^{1,2,3}$

Sempre que houver uso da força ou poder, ameaça contra si próprio ou contra alguém, e que resulte em agravo, lesão ou danos psicológicos é considerado violência. A OMS classificou a violência contra a criança e adolescente em quatro tipos, abuso físico, sexual, emocional ou psicológico e negligência, sendo que esses fatores podem resultar em danos físicos, psicológicos, prejuízo no crescimento, desenvolvimento e maturação das crianças. ${ }^{4}$ O Ministério da Saúde no Brasil possui um detalhamento minucioso acerca das práticas definidas como violência sexual. ${ }^{5}$

A partir da década de 80, através da Constituição da República Federativa do Brasil e do Estatuto da Criança e do Adolescente (ECA), tornou-se obrigatória a notificação de casos confirmados ou de suspeita de atos de violência sexual, penalizando qualquer pessoa que omita alguma informação, afirmando que todas as crianças e adolescentes são sujeitos e que assim como qualquer cidadão, possui seus direitos na sociedade. Há alguns anos, todo menor era considerado juridicamente como objeto pertencente aos interesses dos adultos, e com a nova legislação, as crianças e adolescentes passam a ser considerados como um ser humano portador de direitos, desejos e vontades próprias. ${ }^{6,7}$

A violência sexual agrega em todos os contextos socioculturais em diversas partes do mundo a provocação da população para a notificação destes casos, e na busca de novos casos, com o intuito de aplicabilidade da lei e punição aos agressores, sendo também vista como um desafio social, pois esta experiência traumática na infância ou adolescência reflete em atos para a vida toda, como déficits 
emocionais, comportamentos impulsivos, transtornos mentais, abuso de substâncias ilícitas, considerados pela sociedade como agentes transgressores dos direitos da infância. ${ }^{7-9}$

Após a criação do ECA, surge um grande aliado para assegurar os direitos das crianças e adolescentes, que foi o Conselho Tutelar, o qual coube a missão de fazer cumprir a lei prevista, por meio de mecanismos diversos, porém estruturado, pois não há uma prescrição de como o conselheiro deve agir, mas o mesmo deve estar capacitado para interagir com a criança ou adolescente agredida, assumindo uma postura interdisciplinar e com o olhar voltado a toda família. Desta forma torna-se imprescindível que os profissionais que realizam o atendimento à vítima estejam capacitados para tal intervenção, uma vez que, a falta de preparo dos profissionais como um todo é considerado um obstáculo para obter-se a informação correta, além da escassez de ferramentas de notificações, totalizando um número indescritível de subnotificações ou dados incompletos de violência sexual. ${ }^{10-}$ 12

A OMS estima que 150 milhões de crianças no mundo com idade de 0 a 18 anos sofrem violência sexual, de forma direta ou indireta e de todas as formas, sendo considerado um fenômeno social, que abrange todas as classes, faixa etária e gênero. Um estudo na Tanzânia reconheceu um aumento significativo de casos de violência sexual contra crianças e adolescentes. No mundo a prevalência de violência sexual infantil varia entre 2 e $62 \%$ para meninas e de 3 a $16 \%$ para meninos, na Tanzânia estes índices atingem 28\% em meninas e 13\% em meninos, tendo um aumento entre 1999 e 2007 de 3.500 casos, sendo a maior prevalência em meninas até 18 anos, residentes em área urbana. Outro estudo aponta que a maior prevalência ocorre em adolescentes em idade reprodutiva e de classe média baixa. ${ }^{13-16}$

Na Ásia e Oceano pacífico, autores conduziram uma metanálise para estimar a prevalência, incidência e consequências da violência sexual contra crianças e adolescentes, e os dados mostraram que as crianças violentadas apresentavam maior risco para desenvolver doenças físicas e mentais, ideias suicidas, além de se tornarem prováveis agressores no futuro. Inúmeras pesquisas relacionadas à prevalência, de violência sexual, ocorrem na África, ou países subjacentes e demais países subdesenvolvidos, devido ao fato de que a incidência é maior em mulheres, de classe econômica inferior, onde a vulnerabilidade é maior, tanto em ambiente escolar ou com meninas que são órfãs e moradoras de rua. Na Uganda, país também da África do Sul, o estudo nos mostrou a realidade da prática de promoção da saúde, empoderamento do cidadão e práticas que nos reportam à época da Polícia Médica e educação vulnerável, primando pela segurança e cuidado de toda a população. ${ }^{17-19}$

No Brasil, estima-se que 165 crianças sofrem violência sexual por dia. Entretanto estes são dados parciais devido à falta de fidedignidade das informações pela subnotificação de ocorrências. Os dados sócio demográficos mostram que a grande maioria dos atos de violência sexual ocorrem na residência destas crianças $(64,5 \%)$. Em $45,6 \%$ dos casos o provável autor da violência era do sexo 
masculino. Grande parte dos agressores são pais e outros familiares, ou alguém do convívio muito próximo da criança e do adolescente, como amigos e vizinhos. No estado de Santa Catarina, ,foram registradas 104 ocorrências de violência sexual no período de dois anos, entre 2006 a 2008, o que representa $21 \%$ das mais de 20 mil demandas relacionadas a violações de direitos da população infanto-juvenil, registradas entre janeiro e março de 2015. , 10, $12^{2}$

Avaliando os dados de cidades brasileiras e um breve panorama da prevalência de algumas variáveis referentes à violência infantil, desta forma o objetivo deste este artigo é caracterizar o perfil da violência sexual contra crianças e adolescentes no estado Rio Grande do Sul.

\section{MÉTODO}

Trata-se de um estudo do tipo ecológico, onde foram incluídos dados de notificações do SINAN do período de 2011 a 2015, acessados através do DATASUS. Foram incluídos no estudo os casos de violência sexual notificados no estado do RS, entre crianças e adolescentes (1 a 19 anos), de ambos os sexos.

Foram investigadas variáveis disponibilizadas na base de dados, como faixa etária, sexo, perfil do agressor e local de ocorrência. Os resultados foram apresentados em tabelas descrevendo números absolutos e percentuais.

\section{RESULTADOS}

O total de notificações no período do estudo foi de 6.315. Dentre as vítimas 39,35\% tinham entre 10 e 14 anos, seguido de 30,64\% entre 5 a 9 anos de idade, sendo que a faixa etária que menos sofreu violência sexual foram os menores de 1 ano de idade. Referente ao sexo das vítimas, houve predominância no sexo feminino, totalizando 4.999 (79,16\%) (Tabela 1).

Em relação ao perfil do agressor, podemos observar que entre familiares o padrasto e o pai são os perfis mais comuns ao violentarem sexualmente as crianças e adolescentes, com uma frequência de $14,53 \%$ e $15,74 \%$, respectivamente de. Amigos e conhecidos apontam como o perfil mais comum entre toda a amostra $(25,60 \%)$, seguido dos desconhecidos $(10,43 \%)$.

Da amostra de 6.315 crianças e adolescentes, 74,01\% foram violentadas em suas residências. O segundo local mais prevalente foi a via pública com $8,12 \%$ e posteriormente o ambiente escolar com $2,29 \%$.

Tabela 1: Número de crianças e adolescentes agredidas sexualmente segundo a faixa etária, sexo, perfil do agressor e o local de ocorrência da violência 


\begin{tabular}{lrr}
\hline \multicolumn{1}{c}{ Características } & N & \% \\
\hline Faixa etária & & \\
$<1$ & 99 & 1,56 \\
$1-4$ & 894 & 14,15 \\
$5-9$ & 1935 & 30,64 \\
$10-14$ & 2485 & 39,35 \\
$15-19$ & 902 & 14,28 \\
Sexo & & \\
Masculino & 1316 & 20,83 \\
Feminino & 4999 & 79,16 \\
Perfil do agressor & & \\
Pai & 918 & 14,53 \\
Mãe & 224 & 3,54 \\
Padrasto & 994 & 15,74 \\
Madrasta & 15 & 0,23 \\
Cônjuge & 16 & 0,25 \\
Namorado & 137 & 2,16 \\
Irmão & 235 & 3,12 \\
Amigos ou conhecidos & 1617 & 25,60 \\
Desconhecidos & 659 & 10,43 \\
Cuidadores & 95 & 1,50 \\
Local da agressão & & \\
Residência & 4674 & 74,01 \\
Escola & 145 & 2,30 \\
Bar ou similar & 48 & 0,76 \\
Via pública & 513 & 8,12 \\
Indústrias ou construção & 5 & 0,08 \\
\hline & & \\
\hline
\end{tabular}

\section{DISCUSSÃO}

Este estudo possibilitou uma caracterização do perfil da violência sexual sob o olhar de quatro variáveis quantitativas no estado do RS, no período de 2010 a 2015. Sabe se que a violência sexual é um problema de saúde pública não só no RS, mas como em todo o mundo, principalmente nos países subdesenvolvidos localizados na América do Sul, Ásia e África. ${ }^{3,14,17}$

Através da análise de nossos resultados, podemos verificar que na realidade do RS, a faixa etária mais acometida está entre 10 e 14 anos. Estudo realizado por Frank et al (2010) sugere que esta faixa etária é mais acometida por que engloba um grupo de meninas em fase reprodutiva, ou seja, há uma alta incidência de "meninas mulheres" sendo abusadas por já reproduzirem-se. ${ }^{12}$ No Brasil, essa faixa etária de vítimas, é considerada altamente vulnerável, seguido das vítimas de 5 a 9 anos como na maioria de outros estados ou países. O sexo predominante é o feminino, como aparecem em vários estudos de caracterização do perfil da violência sexual descritos na literatura. ${ }^{1,14,16}$

$\mathrm{Na}$ faixa etária de crianças até nove anos, é comum aparecer abuso sexual sem penetração propriamente dita, pelo fato dos agressores serem membros da família da criança, sendo majoritariamente o pai e o padrasto. A transformação das relações familiares e a falta de relações afetivas atualmente nos reportam para uma sociedade degradada de valores, onde a agressão física e a violência sexual são vistas como um acontecimento comum, porém não normal. Isto justifica o fato de que muitas vezes o perpetrador afirma ter sido ele seduzido pela criança, enfatizando o poder 
masculino. $^{2,20}$ No presente estudo observamos que entre familiares o padrasto e o pai foram os principais agressores.

Em países da África e da Ásia, não diferente do norte e nordeste do Brasil, meninas que moram na rua, são violentadas sexualmente em via pública frequentemente em um percentual inferior às violentadas em sua casa, devido ao fato de que os agressores do sexo masculino sentem-se no direito de intimidar e manter relações tratando as mesmas como suas propriedades. Estes resultados sugerem que há uma destruição do valor familiar e que este pode estrar diretamente relacionado ao abuso sexual infantil e a falta de respeito com as crianças e adolescentes. ${ }^{19,21}$

A maioria dos casos como mostram os dados ocorrem em ambiente domiciliar das vítimas, não somente tendo sido por familiares, mas principalmente por amigos e conhecidos, como tios, padrinhos, vizinhos entre outros. No RS, esse índice de crianças e adolescentes que são violentadas em casa chega a 74,01\%. A omissão destes fatos, ainda é o maior problema para detectarmos a prevalência real de casos notificados. Estima-se que o número de ocorrências seja ainda maior, sendo um problema do estado e de saúde pública. Diversas vezes esta omissão é dos próprios familiares, e isso ocorre por inúmeras situações. Desta forma, todos são responsáveis pela notificação, família, escola, o profissional de saúde, principalmente o pediatra ao realizar a anamnese e o exame físico desta criança, quebrando a barreira do silêncio e protegendo as crianças e adolescentes desta vulnerabilidade à violência sexual infantil..$^{7,8,10}$

Este estudo demonstrou que a faixa etária mais acometida, são as crianças que têm entre 10 a 14 anos, sendo o sexo feminino predominante, além de constatar que, amigos e/ou conhecidos é o perfil mais evidente, sendo a casa da vítima, o local de maior ocorrência do ato de violência sexual infanto juvenil no estado do RS. A violência sexual infanto-juvenil é um problema alarmante amplo e complexo em todos os países do mundo, pelo fato da subnotificação dos casos e pela dificuldade de abordagem com a família e principalmente com a criança. Desta forma, é de vital importância trabalhar as consequências deste ato, sendo que não existe distinção entre idade, gênero e condição familiar. Finalmente, todos os países deveriam estudar a prevalência de tal violência, a fim de gerir mais eficazmente as medidas preventivas, a fim de avaliar qual delas vai ser mais efetiva para a população. O abuso sexual é um problema que diz respeito a todos e, portanto, deve ser intrínseca a iniciativa de contribuir para a luta contra este tipo de abuso em crianças e adolescentes. ${ }^{22-24}$

\section{REFERÊNCIAS}

1 Blake, Márcia de Toledo et al. Characteristics of sexual violence against adolescent girls and adult women. BMC Women's Health.14:15. 2014. DOI: 10.1186/1472-6874-14-15. 
2 Júnior, Antônio A. P.; Borges, Vicente, C.; Santos, Janielly G. Caracterização da violência doméstica contra crianças e adolescentes e as estratégias interventivas em um município do Estado do Rio de Janeiro. Caderno de Saúde coletiva. Rio de Janeiro, 23 (2): 124/131, 2015.

DOI: $10.1590 / 1414-462 X 201500020062$

3 Vieira, Luiza Jane E. S.; Silva, Raimunda M. S.; Cavalcanti, Ludmila F.; Deslandes, Suely Ferreira. Capacitação para o enfrentamento da violência sexual contra crianças e adolescentes em quatro capitais brasileiras. Ciência e Saúde coletiva 20 (11) 3407/3416. 2014. DOI: 10.1590/1413$\underline{812320152011.20512014}$

4 World Health Organization (WHO). World report on violence and health. Geneva: WHO; 2002.

5 World Health Organization (WHO). Preventing child maltreatment: a guide to taking action and generating evidence. Geneva: WHO; 2006.

6 Brasil. Secretaria Especial dos Direitos Humanos da Presidência da República. Programa Nacional de Direitos Humanos. Lei Federal n. 8.069 (Estatuto da Criança e do Adolescente) Brasília. 1990.

7 Zambon, M. P. et al. Violência doméstica contra crianças e adolescentes: um desafio. Rev. Ass. Médica Brasileira. 58 (4):465/471. Campinas/ SP, 2012. http://dx.doi.org/10.1590/S0104$\underline{42302012000400018}$

8 Oliveira, Jaqueline Reiter et al. Violência sexual e coocorrências em crianças e adolescentes: estudo das incidências ao longo de uma década. Ciência e Saúde coletiva. 19 (3) 759/771. 2014. http://dx.doi.org/10.1590/1413-81232014193.18332013

9 Sell, Mariléia; Ostermann, Ana Cristina. A construção da significação das experiências do abuso sexual infantil através da narrativa: uma perspectiva interacional. D.E.L.T.A., 31 (2), (307/332). 2015. http://dx.doi.org/10.1590/0102-445088814836814166.

10 Espindola, Glauco A.; Batista, Vanderléia. Abuso Sexual Infanto Juvenil: A atuação do Programa Sentinela na cidade de Blumenau/ SC. Psicologia, Ciência e Profissão, 33(3), 596/611.SC, Blumenau, 2013. http://dx.doi.org/10.1590/S1414-98932013000300007

11 Hohendorff, Jean Von \& Koller, Sílvia Helena; Habigzang, Luisa Fernanda. Psicoterapia para crianças e adolescentes vítimas de violência sexual no sistema público: Panorama e Alternativas de Atendimento. Psicologia, ciência e profissão 35 (1) 182/198. 2015. http://dx.doi.org/10.1590/1982$\underline{3703000202014}$

12 Frank, Stefanie; Coelho, Elza Berger Salema; Boing, Antonio Fernando. Perfil dos estudos sobre violência contra a mulher por parceiro íntimo: 2003 a 2007. Rev Panamericana de Salud Publica, Washington, v. 27, n. 5, p. 376-381, May 2010. http://dx.doi.org/10.1590/S102049892010000500008

13 Abeid, Muzdalifat et al. Knowledge and atitude towards rape and child sexual abuse, a community based cross sectional study in Rural Tanzânia. BMC Public Health. 2015. DOI: 10.1186/s12889-0151757-7 
14 Decker, Michele R. et al. Prevalence and Health Impact of Intimate Partner Violence and non partner sexual violence among female adolescents aged 15/19 years in urban environment: A multy Country Study. Journal of Adolescents Health. 55, 558/567. 2014. DOI: 10.1016/j.jadohealth.2014.08.022

15 Lundgren, Rebecka; Amin, Avni. Adressing Intimate Partner Violence and Sexual Violence Among Adolescents: Emerging, evidence of effectiveness. Journal of Adolescentes Health 56 (542/550). 2014. DOI: 10.1016/j.jadohealth

16 Trindade, Luciana C. et al. Sexual violence against children and vulnerability. Rev. Ass. Médica Brasileira. 60 (1): 70/74. 2014. http://dx.doi.org/10.1590/1806-9282.60.01.015

17 Devries, Karen M. et al. School violence, mental health, and educational performance in Uganda. Pediatrics, vol. 133,number 1. January, 2014. DOI:10.1542/peds.2013-2007

18 Fang, Xiangming et al. The burden of child maltreatment in the East Asia and Pacific region. Child Abuse Negligence 42 (146/162); Abril, 2015. DOI: 10.1016/j.chiabu.2015.02.012

19 Yahaya, Ismail; Uthman, Olalekan A.; Soares, Joaquim; Macassa, Glória. Social disorganization and history of child sexual abuse against girls in sub Saharan Africa: a multilevel analysis. BMC:International Health \& Human Rights. 2013. DOI: 10.1186/1472-698X-13-33

20 Zuquete, José Gonçalo Pais E. S.; Noronha, Ceci Vilar. “Foi normal, não foi forçado!” versus “Fui abusada sexualmente": uma interpretação dos discursos de agressores sexuais, das suas vítimas e de testemunhas. Physis Revista de Saúde Coletiva. Rio de Janeiro, 22 (4): 1357/1376, 2012. http://dx.doi.org/10.1590/S0103-73312012000400006

21 Mandalazi, P.; Banda, C.; Umar, E.; Street children's vulnerability to HIV and sexually transmitted infections in Malawian cities. Malawi Medical Journal; 25 (1): 1/4 March. 2013.

22 Acunanavas, María José. Abuso sexual en menores de edad: generalidades, consecuencias y prevención. Med. leg. Costa Rica [online]. $31 \quad$ (1) 2014. https://doaj.org/article/9e2cc43b924d48bc87c95cdd900b3c7b

23 Rates, Susana Maria Moreira et al., Violence against children: an analysis of mandatory reporting of violence, Brazil 2011. Ciênc. saúde coletiva, Rio de Janeiro. 20, (3), p. 655-665, Mar. 2015. http://dx.doi.org/10.1590/1413-81232015203.15242014

24 Reynaert, M.. Sexual abuse of children as a form of power abuse and abuse of the body. Acta theologica. 35, p.189-200. 2015. http://dx.doi.org/10.4314/actat.v35i1.11. 


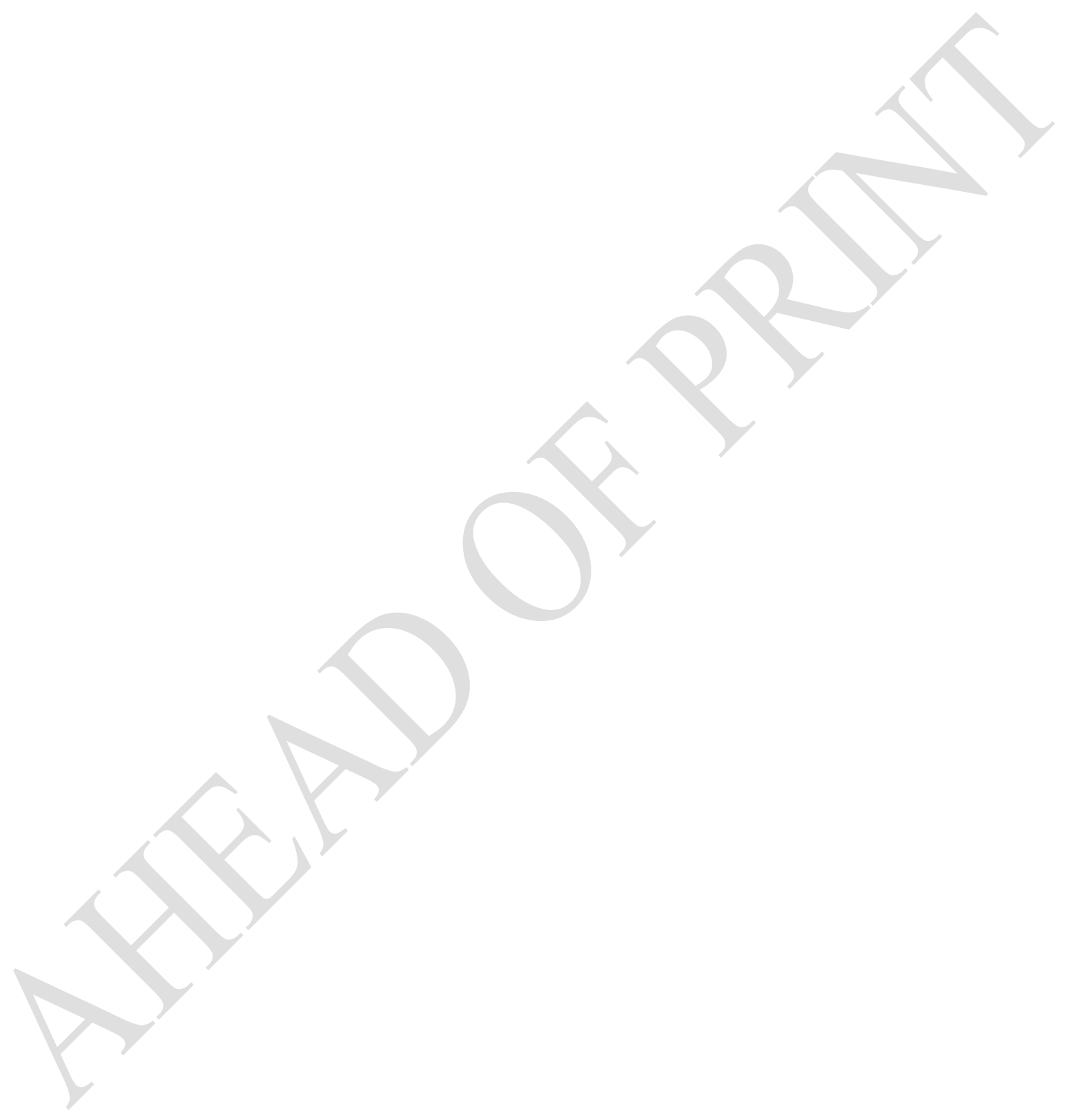

